BULLETIN OF THE

AMERICAN MATHEMATICAL SOCIETY

Volume 79, Number 4, July 1973

\title{
INCIDENCE ALGEBRAS AS ALGEBRAS OF ENDOMORPHISMS
}

\author{
BY WILLIAM GRAVES AND SUZANNE MOLNAR \\ Communicated by Gian-Carlo Rota, December 18, 1972
}

1. Introduction. The order filters on a locally finite partially ordered set $P$ constitute the open sets for a topology on $P$. A sheaf of abelian topological groups will be constructed on the topological space $P$, and the endomorphism ring of this sheaf will be proved isomorphic to the incidence algebra of $P$ (over $Z$ ).

2. A sheaf of abelian groups on $P$. Let $P$ be a locally finite (every interval $[x, y]$ of $P$ is finite) partially ordered set. An (order) filter on $P$ is a subset $V$ of $P$ which contains $y$ whenever $x \leqq y$ and $x \in V$. For $x \in P$,

$$
V_{x}=\{y \in P: x \leqq y\}
$$

is the principal filter generated by $x$. The filters on $P$ are easily seen to be the open sets for a topology on $P$, and the increasing maps from $P$ to another locally finite partially ordered set $Q$ are precisely the continuous functions from $P$ to $Q[5]$.

For each filter $V$ on $P$, let $M(P, V)$, or simply $M(V)$ when reference to $P$ is understood, denote the free abelian group on $V$. For filters $U \subseteq V$, let $r(V, U): M(V) \rightarrow M(U)$ be the group homomorphism determined by

$$
\begin{array}{ll}
x \mapsto x & \text { if } x \in U, \\
x \mapsto 0 & \text { if } x \notin U,
\end{array}
$$

for $x \in V$.

Proposition 1. $M$ (with the restriction maps $r(V, U)$ ) is a sheaf of abelian groups on $P$.

Proof. $M$ is easily seen to be a presheaf of abelian groups. For any open cover $V=\bigcup V_{i}$, consider

$$
M(V) \stackrel{\pi}{\rightarrow} \prod M\left(V_{i}\right) \underset{\pi_{2}}{\stackrel{\pi_{1}}{\rightrightarrows}} \prod M\left(V_{k} \cap V_{j}\right)
$$

where $\pi$ is induced by the restrictions $r\left(V, V_{i}\right), \pi_{1}$ is induced by the restrictions $r\left(V_{k}, V_{k} \cap V_{j}\right)$, and $\pi_{2}$ is induced by the restrictions $r\left(V_{j}, V_{k} \cap V_{j}\right)$. That $\pi$ is injective is clear. Let $\alpha=\left(\alpha_{i}\right) \in \prod M\left(V_{i}\right)$ where $\alpha_{i}=\sum \alpha_{i, x} x$, and suppose that $\pi_{1}(\alpha)=\pi_{2}(\alpha)$. Then $\alpha_{k, x}=\alpha_{j, x}$ for any $x \in V_{k} \cap V_{j}$. So

AMS (MOS) subject classifications (1970). Primary 06A10; Secondary 18F20. 
$\pi(\beta)=\alpha$ where $\beta=\sum \beta_{x} x$ and $\beta_{x}=\alpha_{i, x}$ for any $i$ such that $x \in V_{i}$. Hence, $M$ is a sheaf.

For each filter $V$ of $P$, let $A(V)$ be the commutative ring with basis $V$ of orthogonal idempotents. So $A(V)=M(V)$ as abelian groups, and $A(P)$ is the Möbius algebra (over $Z$ ) studied in [2], [3], [4], [7], and [8]. It is easy to see that the restriction maps introduced above are ring homomorphisms.

COROLlaRY 1. A (with the restriction maps $r(V, U)$ ) is a sheaf of rings on $P$.

An endomorphism, $T$, of the sheaf $M$ is a natural transformation of $M$ considered as a contravariant functor. That is, $T=\{T(V)\}$ consists of group homomorphisms $T(V): M(V) \rightarrow M(V)$, one for each filter $V$ on $P$, which commute with the restriction maps $r(V, U)$.

If $x \leq y$ in $P$, then $r\left(P, V_{x}\right)(y)=0$. So if $T(P)(y)=\sum T(P)(x, y) x$, then it follows from $r\left(P, V_{x}\right) \circ T(P)=T\left(V_{x}\right) \circ r\left(P, V_{x}\right)$ that $T(P)(x, y)=0$ if $x \leq y$. It now follows easily that

THEOREM 1. The association $T \mapsto T(P)$ is an injective ring homomorphism of the endomorphism ring, $\operatorname{End}(M)$, of the sheaf $M$ to the incidence algebra, $\mathscr{I}(P)$, of $P$ (over $Z$ ).

$P$ is lower finite if for each $y \in P$, the set $\{x \in P: x \leqq y\}$ is finite.

Corollary 2. $\operatorname{End}(M) \simeq \mathscr{I}(P)$ if and only if $P$ is lower finite.

3. A topology on $M(P)$. Subsets $S$ of $P$ satisfying

$$
\{x \leqq y: x \notin S\} \text { is finite for every } y \in P
$$

will be called $(*)$-sets. In particular, every cofinite subset of $P$ is a $(*)$-set. For each $(*)$-set $S$, let $N(S)$ be the subgroup of $M(P)$ generated by $S$. Since $S \cap R$ is a (*)-set whenever both of $S$ and $R$ are, $N(S) \cap N(R)=N(S \cap R)$. So the collection $\mathscr{B}=\{N(S): S$ is a $(*)$-set $\}$ is a base for a filter of neighborhoods of 0 determining the structure of abelian topological group on $M(P) \quad[1$, III. 1.2].

PROPOSITION 2. This topology on $M(P)$ is discrete if and only if $P$ is lower finite.

Proof. This topology on $M(P)$ is discrete if and only if $\{0\} \in \mathscr{B}$ if and only if $\varnothing$ is a (*)-set if and only if $P$ is lower finite.

Proposition 3. This topology on $M(P)$ is Hausdorff.

Proof. For $x \in P$, let $S_{x}=P-\{x\}$. Then $S_{x}$ is a .(*)-set, and $\bigcap N\left(S_{x}\right)$ $=\{0\}$. 
A subset $R$ of $P$ is said to be bounded above finitely provided there exists a finite subset $F$ of $P$ such that $x \in R$ implies $x \leqq y$ for some $y \in F$.

Proposition 4. $P$ is bounded above finitely if and only if every (*)-set is cofinite.

Proof. Suppose $P$ is bounded above finitely by the finite subset $F$. For each (*)-set $S$ and each $y \in F$, the set $\{x \leqq y: x \notin S\}$ must be finite. Hence, $S$ is the complement of a finite subset of $P$.

Conversely, assume that every $(*)$-set is cofinite. Then $V_{x}$ is finite for every $x \in P$ since by local finiteness the complement of $V_{x}$ is a $(*)$-set. So $P$ is upper finite and admits an antichain $U$ such that for every $x \in P$ there exists $y \in U$ with $x \leqq y$. But the complement of $U$ is a $(*)$-set. Hence $U$ is finite.

Let $\hat{M}(P)$ be the completion of $M(P)$ as abelian topological group. The summability [1, III. 5.2] in $\hat{M}(P)$ of families $\left\{\alpha_{x} x: x \in P, \alpha_{x} \in Z\right\}$ will be discussed.

THEOREM 2. If $\left\{x: \alpha_{x} \neq 0\right\}$ is bounded above finitely, then $\sum \alpha_{x} x$ exists in $\hat{M}(P)$.

PRoOF. Let $R=\left\{x: \alpha_{x} \neq 0\right\}$. It suffices [1, III. 5.2] to find, for every (*)-set $S$, a finite subset $J$ of $R$ such that $R-J \subseteq S$. But there is a finite subset $F$ of $P$ such that $x \in R$ implies $x \leqq y$ for some $y \in F$. Set

$$
J=\{x \in R: x \notin S\}
$$

which is finite since, for each $y \in F,\{x \leqq y: x \notin S\}$ is finite.

Proposition 5. If $\sum \alpha_{x} x$ exists in $\hat{M}(P)$, then for each $y \in P$ there are at most finitely many $x \geqq y$ with $\alpha_{x} \neq 0$.

Proof. For each $y \in P$, the complement of $V_{y}$ is a (*)-set.

The converse to Proposition 5 is not true. Let $N$ denote the negative integers in their natural order. For each positive integer $i$, let $P_{i}=N$, and let $P$ be the disjoint union of $\left\{P_{i}\right\}$ with the induced order. That is, $P$ is the coproduct in the category of partially ordered sets and increasing maps of the family $\left\{P_{i}\right\}$. For each $x \in P$, let $\alpha_{x}$ be 1 or 0 according as $x=-1 \in P_{i}$ for some $i$ or not. Then for each $y \in P$ there is exactly one $x \in P$ with $x \geqq y$ and $\alpha_{x} \neq 0$. But $\sum \alpha_{x} x$ does not converge since

$$
\left\{x \in P: x \leqq-2 \text { in some } P_{i}\right\}
$$

is a (*)-set which does not meet $\left\{x \in P: \alpha_{x} \neq 0\right\}$.

CONJECTURE. $\sum \alpha_{x} x$ exists if and only if $\left\{x: \alpha_{x} \neq 0\right\}$ is bounded above finitely. 
Let $\tilde{M}(P)$ be the subgroup of $\hat{M}(P)$ consisting of all sums of all families $\left\{\alpha_{x} x: x \in P, \alpha_{x} \in \boldsymbol{Z}\right\}$ such that $\left\{x: \alpha_{x} \neq 0\right\}$ is bounded above finitely. So $M(P) \subseteq \tilde{M}(P) \subseteq \hat{M}(P)$, and, for example, $\sum_{x \leqq y} x$ is in $\tilde{M}(P)$ for each $y \in P$.

4. A sheaf of abelian topological groups on $P$. For each filter $V$ on $P$, $M(V)$ is a subgroup of $M(P)$ and is therefore an abelian topological group (with the subspace topology). Let $\hat{M}(V), \tilde{M}(V)$ be the closures of $M(V)$ in $\hat{M}(P), \tilde{M}(P)$ respectively. It is easy to see that the restriction maps $r(V, U)$ are continuous (in fact, they are strict morphisms, [1, III. 2.8]). Hence, there are induced restriction maps $\tilde{r}(V, U): \tilde{M}(V) \rightarrow \tilde{M}(U)$ for filters $U \subseteq V$ on $P$. It follows that

Proposition 6. $\tilde{M}$ (with the restriction maps $\tilde{r}(V, U)$ ) is a sheaf of abelian topological groups on $P$.

Let $T \in \mathscr{I}(P)$. Consider the association $\sum \alpha_{y} y \rightarrow \sum \alpha_{y} T(x, y) x$. Since $\sum \alpha_{y} y=0$ implies $\alpha_{y}=0$ for all $y$, this association defines a map

$$
\tilde{T}: \tilde{M}(P) \rightarrow \tilde{M}(P) \text {. }
$$

$\widetilde{T}$ is a group homomorphism. To show that $\widetilde{T}$ is continuous, it suffices to show that for any $(*)$-set $S$ there exists a $(*)$-set $S^{\prime}$ such that $\widetilde{T}(x) \in \widetilde{N}(S)$ for every $x \in S^{\prime}(\tilde{N}(S)$ is the closure of $N(S)$ in $\tilde{M}(P))$. But the set $S^{\prime}=\{s \in S: x \leqq s$ implies $x \in S\}$ is a $(*)$-set (by local finiteness) satisfying this condition. From Theorem 1 and topological considerations it then follows that

THeOREM 2. The incidence algebra, $\mathscr{I}(P)$, of $P$ (over $Z$ ) is isomorphic to $\operatorname{End}(\tilde{M})$, the endomorphism ring of the sheaf $\tilde{M}$ of abelian topological groups on $P$.

5. Increasing maps and maps of sheaves. Consider the category whose objects are locally finite partially ordered sets $P$ along with the sheaf $\tilde{M}=\tilde{M}(P, \cdot)$ of abelian topological groups on $P$ and whose morphisms are continuous maps $f: P \rightarrow Q$ (continuous = increasing) along with a morphism $\tilde{f}$ of the sheaf $\tilde{M}(Q, \cdot)$ on $Q$ to the sheaf $\tilde{M}(P, \cdot) \circ f^{-1}$ on $Q$. So, for the increasing map $f: P \rightarrow Q$ and each filter $V$ on $Q$,

$$
\tilde{f}(V): \tilde{M}(Q, V) \rightarrow \tilde{M}\left(P, f^{-1}(V)\right)
$$

is a continuous group homomorphism, and these maps commute with the restriction maps $\tilde{r}(V, U)$ for filters $U \subseteq V$ on $Q$. In particular, for an increasing map $f: P \rightarrow Q$ such that, for each $q \in Q,\{p \in P: f(p)=q\}$ is bounded above finitely, $\tilde{f}$ can be defined as follows: for each filter $V$ on $Q$,

$$
\tilde{f}(V): \tilde{M}(Q, V) \rightarrow \tilde{M}\left(P, f^{-1}(V)\right)
$$


is determined by $q \mapsto \sum_{f(p)=q} p$ for $q \in V$. Many of the results concerning Möbius inversion admit simple proofs in this setting. For example, the following result due to Rota [6] has been elegantly proved by Greene [4] in its finite form in the context of the Möbius algebra and here is proved in its most general form using only the continuous linearity of $\tilde{f}$.

TheOREM 3 (Rota). Let $\sigma: P \rightarrow Q, \tau: Q \rightarrow P$ be increasing maps of locally finite partially ordered sets satisfying

(1) $\tau(\sigma(p)) \geqq p$ for all $p \in P$;

(2) $\sigma(\tau(q)) \leqq q$ for all $q \in Q$.

Then for $p \in P$ and $q \in Q$ with $\sigma(p) \leqq q$,

$$
\begin{aligned}
\sum_{p \leqq x ; \sigma(x)=q} \mu(P)(p, x) & =\sum_{y \leqq q ; \tau(y)=p} \mu(Q)(y, q) & & \text { if } \tau(\sigma(p))=p, \\
& =0 & & \text { if } \tau(\sigma(p))>p .
\end{aligned}
$$

Proof. Here, $\mu(P)$ denotes the Möbius function of $\mathscr{I}(P)$; that is, the (continuous) inverse of the continuous linear automorphism of $\tilde{M}(P)$ determined by $p \mapsto \delta(P, p)=\sum_{x \leqq p} x$ and denoted $\zeta(P)$ (the zeta function of $P$ ). Hence,

$$
p=\sum_{x \leqq p} \mu(P)(x, p) \delta(P, x) .
$$

Since $\sigma(x) \leqq y$ if and only if $x \leqq \tau(y)$, it follows that $\tilde{\sigma}(\delta(Q, q))=\delta(P, \tau(q))$ for any $q \in Q$ where $\tilde{\sigma}=\tilde{\sigma}(P)$. So from (1),

$$
\tilde{\sigma}(q)=\sum_{y \leqq q} \mu(Q)(y, q) \delta(P, \tau(y)) .
$$

But from definition of $\tilde{\sigma}$ and (1),

$$
\tilde{\sigma}(q)=\sum_{p \leqq x ; \sigma(x)=q} \mu(P)(p, x) \delta(P, p) .
$$

The theorem follows from comparison of the coefficients of $\delta(P, p)$ in each of these expressions for $\tilde{\sigma}(q)$.

6. Remarks. Local finiteness was used only in Proposition 4 and was given only to shed light on the concept of $(*)$-set and the related conjecture on summability. So the construction of the sheaf $M$ does not depend on local finiteness. This suggests letting $\operatorname{End}(\tilde{M})$ be the incidence algebra of an arbitrary partially ordered set. This definition and a more detailed account of the results given here will appear later.

The subgroups $N(S), S$ a $(*)$-set, of $M(P)$ are ideals of the Möbius algebra structure $A(P)$ on $M(P)$. Thus, $\tilde{M}$ can be considered as a sheaf $\tilde{A}$ of topological rings, and $\tilde{A}(P)$ is a continuous generalization of the Möbius algebra construction (over $Z$ ). 


\section{REFERENCES}

1. N. Bourbaki, Elements of mathematics. General topology, Addison-Wesley and Hermann, Paris, 1966. MR 34 \# 5044b.

2. R. L. Davis, Order algebras, Bull. Amer. Math. Soc. 76 (1970), 83-87. MR 40 \# 4185.

3. L. Geissinger, Valuations of distributive lattices (to appear).

4. C. Greene, On the Möbius algebra of a partially ordered set (to appear).

5. F. Lorrain, Notes on topological spaces with minimum neighborhoods, Amer. Math. Monthly 76 (1969), 616-627. MR 40 \#1966.

6. G.-C. Rota, On the foundations of combinatorial theory, I. Theory of Möbius functions, Z. Wahrscheinlichkeitstheorie und Verw. Gebiete 2 (1964), 340-368. MR 30 \#4688.

7. - On the combinatorics of the Euler characteristic, Studies in Pure Mathematics (Presented to Richard Rado), Academic Press, London, 1971, pp. 221-233. MR 44 \# 126.

8. L. Solomon, The Burnside algebra of a finite group, J. Combinatorial Theory 2 (1967), 603-615. MR 35 \# 5528.

Department of Mathematics, University of North Carolina, Chapel Hill, North CAROLINA 27514 